\title{
Hypoxia induces autophagy in human vascular endothelial cells in a hypoxia-inducible factor 1-dependent manner
}

\author{
JIANBO WU, ZHEN LEI and JINGUI YU \\ Department of Anesthesiology, Qilu Hospital of Shandong University, Jinan, Shandong 250012, P.R. China
}

Received November 30, 2013; Accepted May 28, 2014

DOI: $10.3892 / \mathrm{mmr} .2014 .3093$

\begin{abstract}
Hypoxia has been widely implicated in numerous pathological conditions, including those associated with inflammation and tumorigenesis. A number of recent studies have implicated hypoxia in the control of microvascular damage, the basis for which is not fully understood. In the present study, it was identified that autophagy was induced in human umbilical vein endothelial cells (HUVECs) post treatment with hypoxia, and the induction of autophagy by hypoxia was enhanced by hypoxia-inducible factor 1 (HIF-1) gene overexpression and inhibited by HIF-1 knockout. Furthermore, the autophagy induced by overexpression of HIF-1 was associated with a reduction of HUVEC cell viability. Therefore, HIF-1 reduced HUVEC cell viability by inducing autophagy. These findings provide evidence of an important link between hypoxia and microvascular damage associated with HIF-1-related autophagy.
\end{abstract}

\section{Introduction}

Hypoxia frequently accompanies various vascular disorders, including thrombosis, atherosclerosis and ischemia/reperfusion injury. Vascular endothelial cells anatomically position at the interface of the blood and tissue exchange, and therefore are frequently exposed to environments of low oxygen tension. The two crucial functions of endothelium, including the maintenance of a permeability barrier and the preservation of the fluidity of blood, are adversely affected by levels of hypoxia that occur in ischemic syndromes $(1,2)$. The impact of hypoxia on endothelial cells is complex and variable. Extended durations of oxygen deprivation may result in endothelial cell death (3-5), while moderate and brief periods of hypoxia in these cells may activate pro-survival or

Correspondence to: Dr Jingui Yu, Department of Anesthesiology, Qilu Hospital of Shandong University, 107 Wenhua Xi Road, Jinan, Shandong 250012, P.R. China

E-mail: jgyusd@163.com

Key words: hypoxia, autophagy, hypoxia-inducible factor 1, human umbilical vein endothelial cells proliferative processes via the hypoxia-induced activation of signaling cascades (6-8).

Hypoxia-inducible factor 1 (HIF-1) is a transcription factor, which functions as a master regulator of adaptive responses to conditions of reduced $\mathrm{O}_{2}$ (9). HIF-1 improves local microcirculation, via its effects on vascular growth and functioning, and regulates $\mathrm{O}_{2}$ utilization by switching oxidative metabolism to glycolytic metabolism (10-12). HIF-1 is composed of HIF- $1 \alpha$ and HIF-1 $\beta$ subunits $(13,14)$. HIF-1 $\alpha$ is the $\mathrm{O}_{2}$-regulated subunit that is specific to HIF-1, whereas HIF-1 $\beta$ is also known as the aryl hydrocarbon receptor nuclear translocator (ARNT) as it is also able to dimerize with the aryl hydrocarbon receptor (15). Hypoxia and reoxygenation potently increase HIF-1 transcriptional activity and HIF- $1 \alpha$ protein expression levels (16-19). Upregulated HIF-1 $\alpha$ activation has been demonstrated in the human heart under conditions of myocardial ischemia and infarction (20), and in patients with coronary artery disease $(21,22)$.

Autophagy, a dynamic catabolic process in which cellular components are delivered to the lysosome for degradation, has been implicated in a wide array of physiological processes and in the pathogenesis of a diverse number of diseases (23-25). Aside from its established roles in homeostasis maintenance and stress adaptation, autophagy also possesses functions in cellular differentiation (26-30). It is a closely regulated process that facilitates the maintenance of a balance among the synthesis, degradation and subsequent recycling of cellular products. However, it remains unclear how hypoxia-induced autophagy is involved in hypertension and coronary disease.

The present study focuses on determining the hypoxia-induced autophagy in human umbilical vein endothelial cells (HUVECs) and further examining the role of autophagy on cell viability. To the best of our knowledge, the results demonstrate novel findings, , that autophagy is an important mechanism of hypoxia-induced HUVEC cell viability reduction that acts via a HIF-1-dependent pathway.

\section{Materials and methods}

Cell culture. HUVECs were cultured in M199 medium (Gibco, Rockville, MD, USA) containing $10 \%$ fetal calf serum (Gibco) and grown on $0.5 \%$ gelatine type A (Sigma-Aldrich, St. Louis, MO, USA) coated T75 dishes in an incubator $\left(5 \% \mathrm{CO}_{2}\right)$. For augmentation, confluent HUVECs were split in $10 \mathrm{~cm}$ dishes using $0.25 \%$ trypsin or trypsin/EDTA. The present study 
was approved by the ethics committee of Qilu Hospital of Shandong University (Shandong, China).

Reagents and antibodies. The Beclin 1 polyclonal antibody was purchased from Santa Cruz Biotechnology, Inc. (Santa Cruz, CA, USA). Autophagy-related gene (Atg)5 and Atg7 monoclonal antibodies were purchased from Cell Signaling Technology Inc. (Danvers, MA, USA). Rapamycin, 3-methyladenine (3-MA) and polyclonal antibodies for GAPDH and LC3-II were purchased from Sigma-Aldrich (St. Louis, MO, USA). The coding sequence of MAP1-LC3 fusion with GFP was synthesized and cloned into the pcDNA3.1 (+) to construct the LC3-GFP-expressing plasmid. The coding sequence of HIF-1 was synthesized and cloned into the pcDNA3.1 (+) to construct the HIF-1-pcDNA3.1-expressing plasmid. HIF-1 siRNA sequence was designed and duplexes were produced by GenePharma, Co. (Shanghai, China).

Quantitative GFP-LC3 analysis and electron microscopy. Quantitative GFP-LC3 light microscopy autophagy assays were performed in the HUVECs with various treatments. HUVECs grown to $80 \%$ confluency were transfected with a GFP-LC3-expressing plasmid, using Lipofectamine 2000 (Invitrogen Life Technologies, Carlsbad, CA, USA). After $24 \mathrm{~h}$ transfection, the cells were subjected to rapamycin treatment $(200 \mathrm{nM})$ for another $24 \mathrm{~h}$ and analyzed by fluorescence microscopy. In another two groups, the cells were pretreated with hypoxia. A total of $2 \mathrm{~h}$ later, the cells were subject to (3MA; $100 \mu \mathrm{M}$ ) for another $24 \mathrm{~h}$ and analyzed by fluorescence microscopy. Electron microscopy was performed to determine the autophagic vacuoles in HUVECs with or without hypoxia treatment. Briefly, HUVEC cell samples with or without hypoxia or rapamycin treatment, were washed three times with $1 \mathrm{X}$ phosphate-buffered saline, trypsinized and collected by centrifuging at $1,000 \mathrm{x} \mathrm{g}$ for $5 \mathrm{~min}$. The cell pellets were fixed with $4 \%$ paraformaldehyde overnight at $4^{\circ} \mathrm{C}$, post-fixed with $1 \% \mathrm{OsO}_{4}$ in cacodylate buffer for $1 \mathrm{~h}$ at room temperature and dehydrated stepwise with ethanol. The dehydrated pellets were rinsed with propyleneoxide for $30 \mathrm{~min}$ at room temperature and then embedded in Spurr resin for sectioning. The images of the thin sections were observed under a transmission electron microscope (JEM1230; JEOL, Ltd, Tokyo, Japan).

RNA isolation and quantitative $(q) P C R$. Total cellular RNA from $2 \times 10^{5}$ to $5 \times 10^{5}$ cells was prepared with TRIzol and reverse transcription (RT) was performed with M-MLV Reverse Transcriptase (Promega Corporation, Madison, WI, USA). Primer sequences and conditions are available upon request. For quantitative analysis of the mRNA expression of Beclin 1, Atg5 and Atg 7, qPCR was conducted by LightCycler 480 (Roche Applied Science, Mannheim, Germany), $1 \mu \mathrm{g}$ RNA per sample was converted to cDNA and used for qPCR. The data were normalized based on GAPDH.

Plasmid overexpression and siRNA knockdown of $H I F-1$. HUVECs in 6-well plates were transfected with HIF-1-pcDNA3.1-expressing plasmid or siRNA duplexes specific for the knockdown of human HIF-1. HIF-1 overexpression or depletion was confirmed by western blot analysis.
Western blot analysis. The cell extracts were prepared following the standard procedures and the proteins were detected by western blotting using polyclonal (human) anti-Beclin 1 antibody, monoclonal (human) anti-Atg5 antibody, monoclonal (human) anti-Atg7 antibody or polyclonal (rabbit) anti-LC3 or GAPDH antibody (Sigma-Aldrich, St. Louis, MO, USA). Goat anti-mouse IgG or goat anti-rabbit IgG (Pierce Biotechnology, Inc., Rockford, IL, USA) secondary antibody conjugated to horseradish peroxidase and ECL detection systems (Super Signal West Femto; Pierce Biotechnology, Inc.) were used for detection.

Cell viability assay. The cell viability was determined by an MTT assay. HUVECs were seeded in 96-well plates and following $24 \mathrm{~h}$, the medium was replaced with $\alpha$-MEM medium containing $1 \%$ FBS. At $0,12,24$ and 48 h post-treatment, the incubation medium in the test wells was replaced with $50 \mu \mathrm{l}$ 1X MTT solution, and the cells were incubated for $2 \mathrm{~h}$ at $37^{\circ} \mathrm{C}$. Following incubation, the MTT solution was discarded and $150 \mu \mathrm{l}$ dimethyl sulfoxide was added to dissolve the precipitate completely at room temperature. The optical density was then measured at $570 \mathrm{~nm}$ using a spectrophotometer. The cell viability was expressed as relative viable cells (\%) to control HUVECs. All of the experiments were performed in three separate experiments.

Statistical evaluation. For GFP-LC3 dot number analysis, the relative mRNA expression of Beclin 1, Atg5 or Atg7 to GAPDH, MTT measurements, statistical evaluations are presented as the mean \pm standard error. The data were analyzed using the Student's t-test. $\mathrm{P}<0.05$ was considered to indicate a statistically significant difference between values.

\section{Results}

Hypoxia induces autophagy and autophagy-associated molecule expression in HUVECs. HUVECs were transfected with GFP-LC3, a biomarker for autophagy, and exposed to hypoxic conditions for $24 \mathrm{~h}$. LC3, a microtubule-associated protein light chain 3 (MAP-LC3), typically exhibits diffuse cytosolic distribution. Representative fluorescence images, as demonstrated in Fig. 1A-C, indicated that the treatment of hypoxia or rapamycin led to the redistribution of LC3 to punctate structures and increased the number of LC3-GFP-positive vesicles in cells. The ultrastructures of HUVECs with or without hypoxic treatment were observed by EM microphotography (Fig. 1D-E). The prominent features of cells subject to hypoxia treatment were observed in the form of autophagic vacuoles and autolysosomes in the cytoplasm.

In addition, it has been demonstrated that LC3-II increases during autophagy compared with LC3-I (31). When autophagy is activated, the LC3-I protein localized in the cytoplasm is cleaved, lipidated and inserted as LC3-II into autophagosome membranes. To detect the expression of LC3-II, western blotting was performed with lysates from HUVECs subject to hypoxia or rapamycin (Fig. 2A). As part of a type III PI3 kinase complex, the autophagy gene Beclin 1 , which is required for the formation of the autophagic vesicles (32). Also, autophagy-related gene (ATG) products have essential roles in autophagy, such as Atg 5 and Atg 7. 

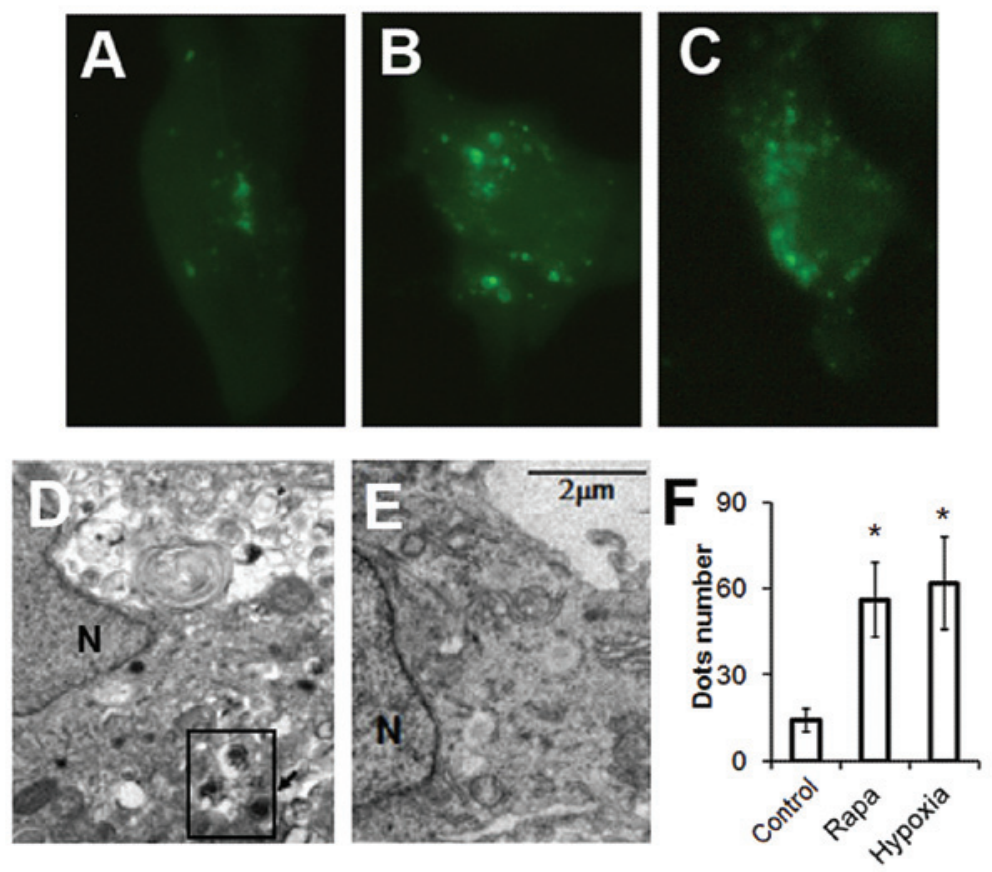

Figure 1. Hypoxia induced autophagy in HUVECs. (A-C and F) HUVECs were transfected with a plasmid that expresses a GFP-LC3 fusion protein. Following $24 \mathrm{~h}$, the cells were incubated for another $24 \mathrm{~h}$ at $37^{\circ} \mathrm{C}$ in Dulbecco's modified Eagle's medium with 1/10,000 dimethyl sulfoxide (control), under hypoxia $\left(1 \% \mathrm{O}_{2}\right)$ or $100 \mathrm{nM}$ Rapa. Following fixation, cells were immediately visualized by fluorescence microscopy. The number of punctate GFP-LC3 dots in each cell was counted and at least 100 cells were included for each group. (D and E) Electron microscopy was performed on HUVECs post hypoxic treatment as described in the Materials and methods. More acidic vesicular organelles were observed in the HUVECs post hypoxic treatment. The data were normally distributed and were statistically analyzed using the Student-Newman-Keuls test. "P $<0.01$, significant difference vs. the control. Rapa, rapamycin; HUVECs, human umbilical vein endothelial cells.

Fig. 2B and $\mathrm{C}$ demonstrates that the expression of Atg 5, Atg 7 and Beclin 1 at an mRNA and protein level increased following hypoxia or rapamycin treatment. These results significantly indicate that hypoxia or rapamycin treatment induces autophagy in HUVECs.

Hypoxia-induced autophagy-related molecule expression in HUVECs may be regulated by HIF-1. To reveal the precise underlying mechanisms behind the above demonstrated activation of autophagy in HUVECs, the alteration of HIF-1 expression with a pcDNA3.1 eukaryotic plasmid and chemical synthesized siRNA was further examined. HIF-1, a key transcription factor, has pivotal roles in hypoxia. The HIF-1 overexpression with eukaryotic plasmid or HIF-1 knockout by siRNA was transfected to the HUVECs. As revealed in Fig. 3A-D, overexpression of HIF-1 significantly improved the occurrence of autophagy, and HIF-1 knockout attenuated the formation of autophagic vacuoles in HUVEC cells. More importantly, HIF-1 overexpression markedly promoted the cleavage of LC3 and expression of Atg5, Atg7 and Beclin1. By contrast, transfection with HIF-1 siRNA, which respectively blocked HIF-1 expression, significantly prevented LC3-II production, as well as the expression of Atg5, Atg7 and Beclin 1 (Fig. 3E and F). Therefore, these data demonstrated that autophagy to hypoxia is dependent on HIF-1 in HUVEC cells. It may therefore be concluded that the HIF-1 pathway regulates autophagy activation in HUVECs under hypoxic conditions.

Decrease in HUVEC cell viability due to hypoxia-induced autophagy is dependent on HIF-1. As the results above indi-
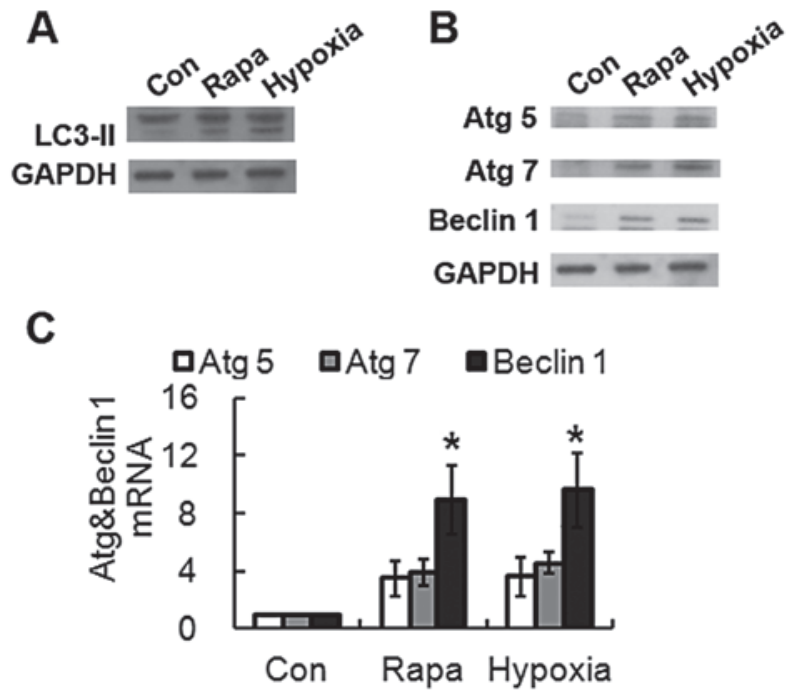

Figure 2. Hypoxia increases the conversion of LC3-I to LC3-II and increases Atg 5, Atg 7 and Beclin 1 expression at an mRNA and protein level. (A and B) Following hypoxia or Rapa treatment for $24 \mathrm{~h}$, the cells were lysed and subjected to western blotting with the antibodies indicated. (C) Atg 5, Atg 7 or Beclin 1 expression in mRNA levels of HUVECs $24 \mathrm{~h}$ post treatment of hypoxia or $100 \mathrm{nM}$ Rapa. The total cell mRNA was analyzed by quantitative polymerase chain reaction. " $\mathrm{P}<0.01$ vs. control. Atg, autophagy-related gene; Rapa, rapamycin.

cated, HIF-1 induced significant autophagy and a high level expression of autophagy-associated molecules in HUVECs. Firstly, western blotting was performed to examine the expression of HIF-1 or LC3-II induced by hypoxic conditions or HIF-1 transfection with a pcDNA plasmid in HUVECs. As 

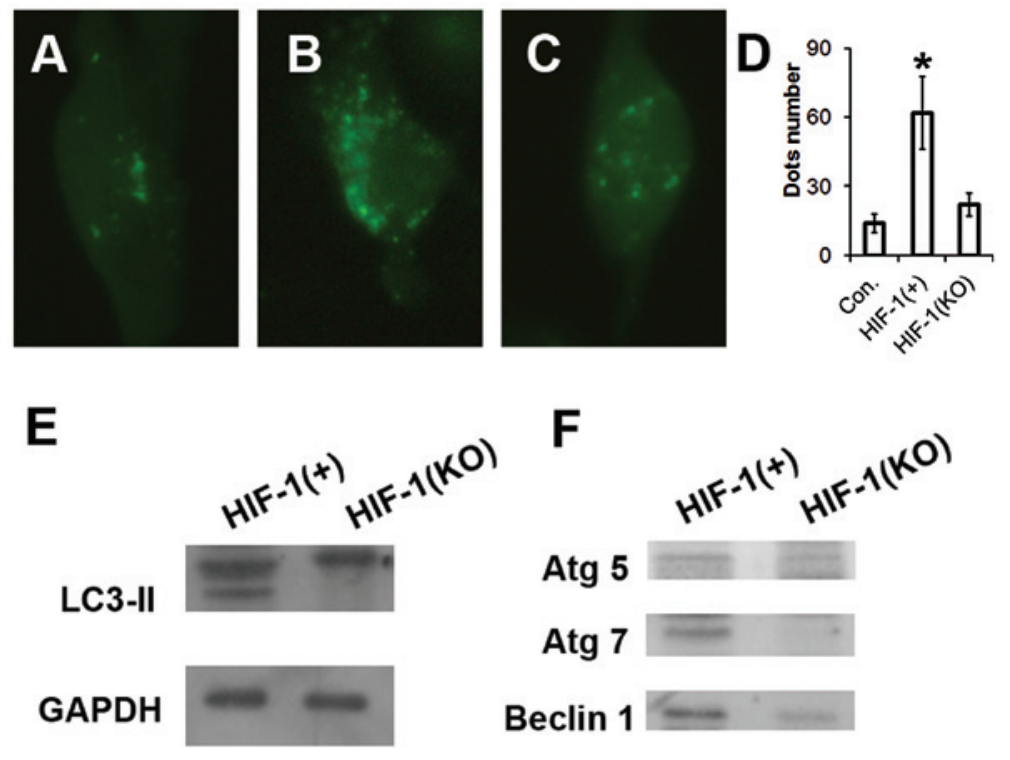

$\mathbf{F}$

Atg 5

Atg 7

Beclin 1
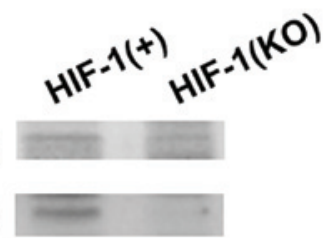

GAPDH

Figure 3. Hypoxia induces autophagy in HUVECs through the HIF-1 pathway. (A, B, C and D) HUVEC cells were transfected with a plasmid that expresses a GFP-LC3 fusion protein. Following $24 \mathrm{~h}$, the cells were incubated for another $24 \mathrm{~h}$ at $37^{\circ} \mathrm{C}$ in Dulbecco's modified Eagle's medium with $1 / 10,000$ dimethyl sulfide (control), pcDNA-HIF-1 or HIF-1 siRNA as described in the Materials and methods. Following fixation, the cells were immediately visualized by fluorescence microscopy. The number of punctate GFP-LC3 dots in each cell was counted and at least 100 cells were included for each group. . $\mathrm{P}<0.01$ vs. control (E and F) Following pcDNA-HIF-1 or HIF-1 siRNA incubation for $24 \mathrm{~h}$, as described in the Materials and methods, the cells were lysed and subjected to western blotting with the antibodies indicated. HIF-1, hypoxia-inducible factor 1; HUVECs, human umbilical vein endothelial cells.
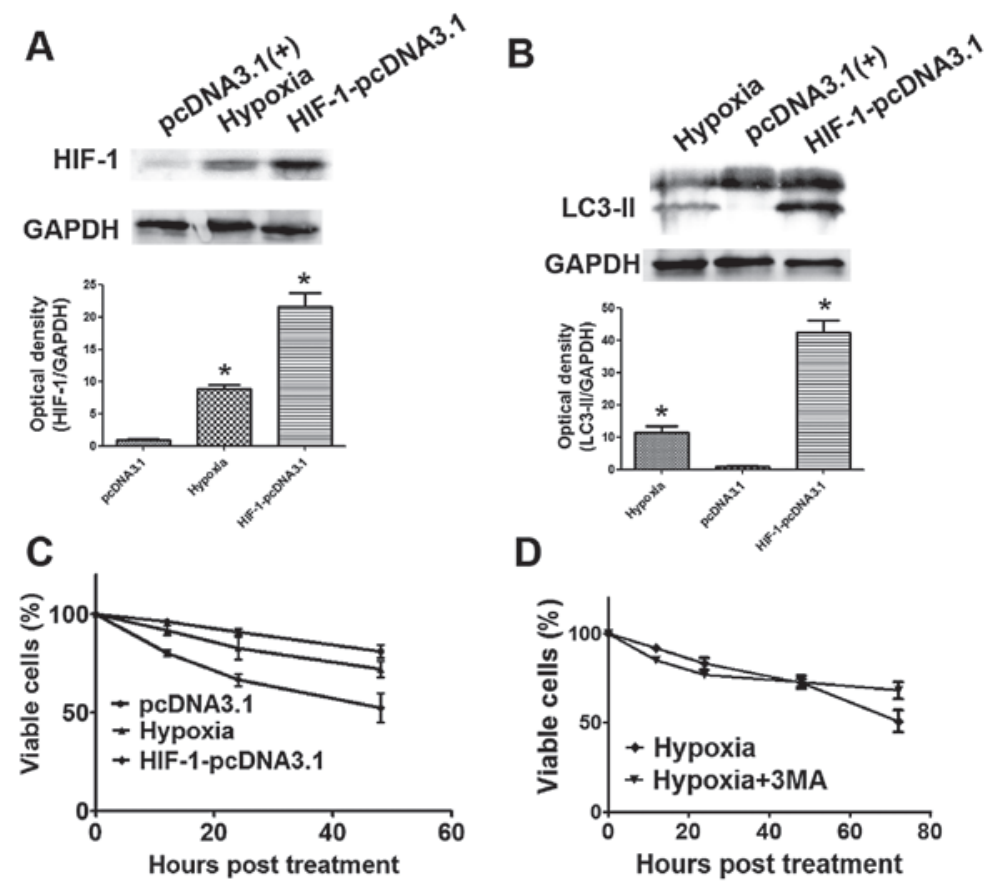

Figure 4. Autophagy induced by high dose of HIF-1 expression reduced the HUVEC cell viability. (A and B) Following pcDNA3.1, pcDNA-HIF-1 transfection or hypoxia treatment of $24 \mathrm{~h}$, as described in the Materials and methods, the cells were lysed and subjected to western blotting with the antibodies indicated. Densitometry was performed for quantification. (C) Viability of HUVEC cells post treatment with pcDNA3.1, pcDNA-HIF-1 transfection or hypoxia treatment. HUVEC cells 12,24 or $48 \mathrm{~h}$ post inoculation with pcDNA3.1, pcDNA-HIF-1 transfection or hypoxia treatment decreased significantly in viability. ${ }^{*} \mathrm{P}<0.05$. (D) 3MA may ameliorate the hypoxia-induced HUVEC cell viability decrease, ${ }^{*} \mathrm{P}<0.05$ vs. pcDNA3.1. HIF-1, hypoxia-inducible factor 1 ; HUVECs, human umbilical vein endothelial cells; 3MA, 3-Methyladenine.

revealed in Fig. 4A-B, the expression of HIF-1 or LC3-II protein stimulated by HIF-1 transfection with a pcDNA plasmid was notably higher than that with hypoxia condition. To further determine the affect of HIF-1 or hypoxia induced autophagy on the cell viability, an MTT assay was conducted to reveal the HUVEC cell viability. It was demonstrated that the cells treated 
with HIF-1 or hypoxia decreased significantly in viability (Fig. 4C). In addition, HIF-1 reduced the cell viability more than hypoxia in the HUVEC cells. Finally, to further confirm the functional significance of HIF-1 in hypoxia-induced cell viability, the HUVEC cells were pretreated with the pharmacological inhibitor of autophagy, 3-MA, followed by exposure to hypoxia. The data revealed that 3-MA treatment evidently prevented viability decreasing in HUVEC cells when cultured under hypoxic conditions (Fig. 4D). In conclusion, the above data suggests that HIF-1 indeed has an important role in the hypoxia-induced reduction in cell viability.

\section{Discussion}

Previous studies have documented that hypoxia-induced cell death is a major concern clinically and has a critical role in various physiological processes, including cell proliferation, hypoxic/ischaemic disease, organ transplantation, angiogenesis, tumor invasion and metastasis (33-36). Clinical studies have demonstrated that autophagy is an intracellular lysosomal degradation process that is characterized by the formation of double-membrane vesicles in cytoplasm. Therefore, autophagy has key physiological cellular functions, including degradation of long-lived proteins, organelle turnover, adaptation to nutrient depletion, extension of lifespan, cellular development, differentiation and anti-aging (37). Consequently, autophagy may preserve cell viability or be a self-destructive process that leads to cell death (38).

In the present study, an increased in autophagic activity in HUVECs induced by hypoxia was detected. HUVECs are widely used as a source of vascular endothelial cells. To the best of our knowledge, this is the first study to report the effects of hypoxia on autophagic cell death in HUVEC cells. Several approaches were adopted to determine autophagy, including fluorescent LC3-GFP dots, electron microscopic imaging and autophagy-associated molecules expression in mRNA and protein levels. HUVEC cells were exposed to hypoxia and an evident increase in the number of autophagic vacuoles and autolysosomes in the cytoplasm was identified, suggesting enhanced autophagy formation. In addition, the occurrence of autophagy was also confirmed with rapamycin treatment (Fig. 1). The determination of further autophagy-associated molecules also confirmed the autophagy induced by hypoxia or rapamycin, as evidenced by the increase in the expression of LC3-II following the treatments, aswell as the enhanced expression of Beclin 1, Atg 5 and Atg 7 in the HUVEC cells post hypoxia or rapamycin treatment at mRNA and protein levels (Fig. 2). In conclusion, autophagy may be induced by hypoxia in HUVEC cells in vitro.

HIF-1 is a transcription factor that is essential in the regulation of gene expression to maintain oxygen homeostasis (39). Firstly, HIF-1 was proved to coordinate adaptive responses to hypoxia at the cellular and systemic levels $(14,40,41)$. Furthermore, HIF-1 has been demonstrated to regulate the expression of hundreds of target genes involved in angiogenesis, erythropoiesis, metabolism, autophagy and other adaptive responses to hypoxia (42). HIF is a heterodimer of an $\alpha$ subunit that is unstable in the presence of relatively high levels of oxygen and a $\beta$-subunit that is not oxygen regulated. To elucidate the mechanisms by which HUVEC cells respond to hypoxia, an HIF-1 overexpression plasmid and HIF-1 siRNA were used and it was demonstrated that hypoxia triggered autophagy in HUVEC cells via the HIF-1 pathway. A significant increase in the autophagy-specific autolysosomes was observed via the LC3-GFP report vector with a fluorescence microscope in HIF-1-treated cells. Coversely, HIF-1 knockout with siRNA treatment in HUVEC cells led to a notable reduction in autolysosome formation. In addition, the cleavage and recruitment of LC3 to autophagosomes, as well as the expression of autophagy-associated molecules were also enhanced by HIF-1 overexpression and attenuated by HIF-1 knockout (Fig. 3). In conclusion, hypoxia-induced autophagy in HUVEC cells was demonstrated to involve the HIF-1 pathway.

Hypoxia treatment or HIF-1 overexpression may induce autophagy, however, their effects on cell viability appeared to be different. Hypoxia appeared to ameliorate the cell viability; however, cell viability was more markedly decrease following HIF-1 stimulation. HIF-1 was able to significantly inhibit the HUVEC cell viability and this effect was reversed by $3 \mathrm{MA}$ (Fig. 4). Therefore, the autophagy induced by HIF-1 was able to destroy cellular components and acted as a self-destructive process that led to cell death. It may therefore be a novel mechanism involved in the decreased proliferation of the HUVEC cells post treatment with HIF-1.

In summary, the present study suggests that hypoxia induces autophagy in HUVEC cells, due to activation of the HIF-1 pathway. HIF-1 treatment was able to induce excess autophagy and reduce the cell viability. Further studies are required to elucidate the mechanisms of hypoxia-induced apoptosis and autophagic cell death, which may facilitate the development of novel methods for the prevention of hypoxic-ischaemic diseases.

\section{Acknowledgements}

This study was supported by the grant from Qilu Hospital of Shandong University (Shandong, China).

\section{References}

1. Stelzner TJ, O'Brien RF, Sato K and Weil JV: Hypoxia-induced increases in pulmonary transvascular protein escape in rats. Modulation by glucocorticoids. J Clin Invest 82: 1840-1847, 1988.

2. Hamer JD, Malone PC and Silver IA: The $\mathrm{PO}_{2}$ in venous valve pockets: its possible bearing on thrombogenesis. Br J Surg 68: 166-170, 1981.

3. Kloner RA, Rude RE, Carlson N, et al: Ultrastructural evidence of microvascular damage and myocardial cell injury after coronary artery occlusion: which comes first? Circulation 62: 945-952, 1980.

4. Lee C, Cheng W, Chang M, et al: Hypoxia-induced apoptosis in endothelial cells and embryonic stem cells. Apoptosis 10: 887-894, 2005.

5. Stempien-Otero A, Karsan A, Cornejo CJ, et al: Mechanisms of hypoxia-induced endothelial cell death. Role of p53 in apoptosis. J Biol Chem 274: 8039-8045, 1999.

6. Lee S, Chen TT, Barber CL, et al: Autocrine VEGF signaling is required for vascular homeostasis. Cell 130: 691-703, 2007.

7. Mogi M, Fukuo K, Yang J, Suhara T and Ogihara T: Hypoxia stimulates release of the soluble form of fas ligand that inhibits endothelial cell apoptosis. Lab Invest 81: 177-184, 2001.

8. Schäfer M, Schäfer C, Ewald N, Piper HM and Noll T: Role of redox signaling in the autonomous proliferative response of endothelial cells to hypoxia. Circ Res 92: 1010-1015, 2003.

9. Semenza GL: Hypoxia-inducible factors in physiology and medicine. Cell 148: 399-408, 2012.

10. Shohet RV and Garcia JA: Keeping the engine primed: HIF factors as key regulators of cardiac metabolism and angiogenesis during ischemia. J Mol Med (Berl) 85: 1309-1315, 2007. 
11. Taylor CT: Mitochondria and cellular oxygen sensing in the HIF pathway. Biochem J 409: 19-26, 2008.

12. Semenza GL: Hypoxia-inducible factor 1: regulator of mitochondrial metabolism and mediator of ischemic preconditioning. Biochim Biophys Acta 1813: 1263-1268, 2011.

13. Wang GL and Semenza GL: Purification and characterization of hypoxia-inducible factor 1. J Biol Chem 270: 1230-1237, 1995.

14. Wang GL, Jiang BH, Rue EA and Semenza GL: Hypoxia-inducible factor 1 is a basic-helix-loop-helix-PAS heterodimer regulated by cellular $\mathrm{O}_{2}$ tension. Proc Natl Acad Sci USA 92: 5510-5514, 1995.

15. Reyes H, Reisz-Porszasz S and Hankinson O: Identification of the Ah receptor nuclear translocator protein (Arnt) as a component of the DNA binding form of the Ah receptor. Science 256: $1193-1195,1992$

16. Cai Z, Manalo DJ, Wei G, et al: Hearts from rodents exposed to intermittent hypoxia or erythropoietin are protected against ischemia-reperfusion injury. Circulation 108: 79-85, 2003.

17. Yuan G, Nanduri J, Bhasker CR, Semenza GL and Prabhakar NR: $\mathrm{Ca}^{2+} /$ calmodulin kinase-dependent activation of hypoxia inducible factor 1 transcriptional activity in cells subjected to intermittent hypoxia. J Biol Chem 280: 4321-4328, 2005.

18. Yuan G, Nanduri J, Khan S, Semenza GL and Prabhakar NR: Induction of HIF-1alpha expression by intermittent hypoxia: involvement of NADPH oxidase, $\mathrm{Ca}^{2+}$ signaling, prolyl hydroxylases, and mTOR. J Cell Physiol 217: 674-685, 2008.

19. Peng YJ, Yuan G, Ramakrishnan D, et al: Heterozygous HIF-1alpha deficiency impairs carotid body-mediated systemic responses and reactive oxygen species generation in mice exposed to intermittent hypoxia. J Physiol 577: 705-716, 2006.

20. Lee SH, Wolf PL, Escudero R, et al: Early expression of angiogenesis factors in acute myocardial ischemia and infarction. N Engl J Med 342: 626-633, 2000.

21. Hlatky MA, Quertermous T, Boothroyd DB, et al: Polymorphisms in hypoxia inducible factor 1 and the initial clinical presentation of coronary disease. American Heart J 154: 1035-1042, 2007

22. Resar JR, Roguin A, Voner J, et al: Hypoxia-inducible factor 1alpha polymorphism and coronary collaterals in patients with ischemic heart disease. Chest 128: 787-791, 2005.

23. Shintani T and Klionsky DJ: Autophagy in health and disease: a double-edged sword. Science 306: 990-995, 2004.

24. Rosenfeldt MT and Ryan KM: The role of autophagy in tumour development and cancer therapy. Expert Rev Mol Med 11: e36, 2009.

25. Todde V, Veenhuis M and van der Klei IJ: Autophagy: principles and significance in health and disease. Biochim Biophys Acta 1792: 3-13, 2009.
26. Boya P, Mellén MA and de la Rosa EJ: How autophagy is related to programmed cell death during the development of the nervous system. Biochem Soc Trans 36: 813-817, 2008.

27. Kundu M, Lindsten T, Yang CY, et al: Ulk1 plays a critical role in the autophagic clearance of mitochondria and ribosomes during reticulocyte maturation. Blood 112: 1493-1502, 2008.

28. Pua HH, Guo J, Komatsu M and He YW: Autophagy Is Essential for Mitochondrial Clearance in Mature T Lymphocytes. JJ Immunol 182: 4046-4055, 2009.

29. Singh R, Xiang Y, Wang Y, et al: Autophagy regulates adipose mass and differentiation in mice. J Clin Invest 119: 3329-3339, 2009.

30. Srinivas V, Bohensky J and Shapiro IM: Autophagy: a new phase in the maturation of growth plate chondrocytes is regulated by HIF, mTOR and AMP kinase. Cells Tissues Organs 189: 88-92, 2009

31. Kabeya Y, Mizushima N, Ueno T, et al: LC3, a mammalian homologue of yeast Apg8p, is localized in autophagosome membranes after processing. EMBO J 19: 5720-5728, 2000.

32. Sun Y and Peng ZL: Programmed cell death and cancer. Postgraduate Med J 85: 134-140, 2009.

33. Volm M and Koomägi R: Hypoxia-inducible factor (HIF-1) and its relationship to apoptosis and proliferation in lung cancer. Anticancer Res 20: 1527-1533, 2000.

34. Rayner BS, Duong TT, Myers SJ and Witting PK: Protective effect of a synthetic anti-oxidant on neuronal cell apoptosis resulting from experimental hypoxia re-oxygenation injury. J Neurochem 97: 211-221, 2006.

35. Bhogal RH, Weston CJ, Curbishley SM, et al: Variable responses of small and large human hepatocytes to hypoxia and hypoxia/reoxygenation (H-R). FEBS Lett 585: 935-941, 2011.

36. Härtel FV, Holl M, Arshad M, et al: Transient hypoxia induces ERK-dependent anti-apoptotic cell survival in endothelial cells. American J Physiol Cell Physiol 298: C1501-C1509, 2010.

37. Mazure NM and Pouysségur J: Hypoxia-induced autophagy: cell death or cell survival? Curr Opin Cell Biol 22: 177-180, 2010.

38. Tsujimoto Y and Shimizu S: Another way to die: autophagic programmed cell death. Cell Death Differ 12 (Suppl 2): 1528-1534, 2005.

39. Semenza GL: HIF-1 and mechanisms of hypoxia sensing. Curr Opin Cell Biol 13: 167-171, 2001.

40. Iyer NV, Kotch LE, Agani F, et al: Cellular and developmental control of $\mathrm{O}_{2}$ homeostasis by hypoxia-inducible factor 1 alpha. Genes Dev 12: 149-162, 1998

41. Yu AY, Shimoda LA, Iyer NV, et al: Impaired physiological responses to chronic hypoxia in mice partially deficient for hypoxia-inducible factor lalpha. J Clin Invest 103: 691-696, 1999.

42. Semenza GL: Regulation of oxygen homeostasis by hypoxia-inducible factor 1. Physiology 24: 97-106, 2009. 